\title{
Node Failure Management to Improve the Performance of Wireless Sensor Networks
}

\author{
Pallavi Katkar a ${ }^{\text {1 }}$, Ashwini Pawar ${ }^{\text {b }}$, Sheetal Zalte ${ }^{\mathrm{c}}$, Suhas Katkar ${ }^{\mathrm{d}}$ \\ ${ }^{a}$ Shivaji University, Kolhapur, India \\ ${ }^{\mathrm{b}}$ Modern College, Pune, India \\ 'Shivaji University, Kolhapur, India \\ ${ }^{d}$ Varroc Engineering, Pune, India
}

\begin{abstract}
A sensor network can be defined an assembly of sensor nodes which associated by all together to complete particular detailed task. These sensor nodes are mostly in huge amounts also compactly installed moreover in the network area or very near to it. Sensor networks can be worked for several sectors such that: environmental monitoring, home, health care, Industries, military, and habitat. Failure of network is unavoidable in wireless sensor networks because of unfriendly location and non-reachable placement. Hence, it is needed that network faults are discovered in time and proper methods are engaged to bear network task. So, it is important to deliver fault forbearing systems for spread sensor applications. Numerous new work in this field yield severely different methodologies to talking the fault tolerance concern in routing. In this propose review and equate present fault tolerant practices to provision for sensor applications.
\end{abstract}

Keywords.Wireless sensor networks, node failure reasons, fault detection approaches, fault management, fault detection and recovery.

\section{Introduction}

A dynamic field of concern for researchers and manufacturing group is Wireless sensor networks (WSNs). A WSN is usually a network of scattered sensor nodes assembling data from the atmosphere to observe the situations of that atmosphere. There are two types of nodes are used for WSN application which are completely depend on the application requirement, static nodes and movable nodes are the categories of the nodes. Static nodes stay fixed and complete to recognizing the events, routing of packets, else perform as destination nodes, although movable nodes travel everywhere in the network to identify network situation and different jobs such as changing dead nodes. Node distribution is depend on application may be physical or random. In physical distribution, the sensors are physically dropped and data is routed with selected paths [1]. The presentation of WSNs completely rest on the assumption that the nodes are linked till the destination node., few nodes those are situated away from the sink node and because of this its require several steps to communicate with neighboring nodes and transfer the date to destination node[2,3]. Restricted resources are the one of the design issues in sensor node.

\footnotetext{
${ }^{1}$ Pallavi Katkar, Shivaji University, Department of Comp.Sci., India Email: pal.katkar@gmail.com
} 
Several sensor nodes might be dying or congested because of shortage of energy resource, have physical loss or atmosphere interfering. The fault of sensor nodes would not disturb the complete performance of the sensor network [4, 5]. Faulty node or multiple nodes may disturb the connectivity and might be the reason for dividing of the network.

A resolution to this issue is to install renewed nodes changing the failed ones which are an expensive and mostly not feasible method. Instead of this routing algorithm it needed to be self management characteristics are self organizing, healing and fault tolerant. The approach to handling the fault is to plan a scheme that is self healing tolerant to initiate with. Self healing is the capability to sustain sensor network's activity lacking any intermission because of sensor nodes failure. So that routing algorithm can manage node failure management and improve the network performance.

\section{Literature Review}

In Maintaining connectivity while keeping complete handling undamaged by with restricted amount of movable nodes are a tough job, particularly while a particular otherwise numerous nodules collapse. The situation is problematic of extreme significance those are taken considered in the literature survey in recent ages. Several explanations require to be suggested concerning in the topic. [6] This work proposed a novel fault finding scheme to resolve fault related issues. This work suggested four methods. With each fault all methods are checked and after that an inquiry message are taken consider to minimize the wrong results. This find failed node and try to replace those with new one. But it not worked link failure and management. [7] Self-Sensor deployment geographically with automatic manner. Algorithm to define re-location of sensors. Algorithm failed to solve to manager node failure management. Rather they replace the nodes with new node. But every time it is not possible to replace nodes. It is most costly and time consuming. This scheme [8] proposed software oriented less overhead fault discovery technique to identify faults in numerous hardware modules. The failure recognition arrangement has remained performed in the SOS kernel of the sensor nodes. Self healing systems support hardware built sensors those are used in electronic devices. But, its failed serve mobile WSN. [9] An approach to quotation such material from a gathering of described facts are stated. Lastly, subsequent the planned method, a programmed fuzzy logic constructed method for failure recognized in LTE networks is scheduled. Its only work in LTE networks. It is not consider mobility as parameter while considering link failure. [10] An innovative method is projected to decrease battery operated intake those are grounded on the self-motivated grouping with the use of neural network. Using Boltzmann concept in neural network, former data distribution sensor nodes generate cluster dynamically. Loads remain correct conferring for the situations and the situation as well improve the competence of the active grouping. This works considered static nodes not mobile nodes.

\section{Faulty Nodes in WSN}

When it comes to faulty nodes in a sensor network, it is necessary to know about faulty node, node failure reasons, faulty node detection, and diagnosis of fault. In the network 
nodes are get fail with the number of reasons. Some of node failure reasons are Power drain, Out of transmission range, Harsh Environment conditions, malicious nodes, Node movement Speed and direction.

\subsection{Faulty Node}

Sensor node remains assumed to be faulty node if the data sent to the destination or the intermediate (neighboring) nodes are present in the network is improper. Due to faulty nodules network faces many issues like network get splits, network performance get degrades and sometimes its cause end of the network.

\subsection{Node Failure Reasons}

So many reasons are there to have faulty nodes in a sensor network but they cannot be predicted exactly because the sensor nodes are deployed in a huge amount. Still some of the node failures reasons which are occur frequently are;

- Power Drain - Wireless sensor network having limited energy resources.

- Out of transmission range - Ehen nodes are mobile nodes are frequently move out of transmission range causes packet loss issues.

- Malicious Node - malicious nodes in network which behaves so, other nodes become unreachable or dead.

- Congestion - Find the malicious nodes in network which behaves so, other nodes become unreachable or dead.

- Node movement speed and direction - If nodes are mobile then mobility of nodes their speed and direction creates issue for network.

\subsection{Fault Detection Approaches}

Fault discovery mostly scheduled as per the category of the application and also which sort of failure, certain existing failure discovery patterns are discoursed. Here classified the present failure recognition methods are: Centralized, Distributed and Cluster

Table1. Fault Detection Approaches

\begin{tabular}{|c|c|c|c|}
\hline Type & Description & Advantage & Disadvantage \\
\hline Centralized & $\begin{array}{l}\text { A geologically or reasonably } \\
\text { integrated sensor node having central } \\
\text { organizer or administrator which is } \\
\text { concern for observing and locating } \\
\text { faulty or disobedience nodes in the } \\
\text { network. }\end{array}$ & $\begin{array}{l}\text { 1. Capable to perform } \\
\text { extended variety of } \\
\text { failure managing } \\
\text { preservation. } \\
\text { 2.Active detection model } \\
\text { 3.Help for route selection }\end{array}$ & $\begin{array}{l}\text { 1. Unlimited resource } \\
\text { required. } \\
\text { 2. More storage required. } \\
\text { 3. Increased network } \\
\text { overhead. } \\
\text { 4. If center node fails whole } \\
\text { system fails. }\end{array}$ \\
\hline Distributed & $\begin{array}{l}\text { Distributed method inspires the } \\
\text { conception of local management, } \\
\text { which uniformly allocates fault } \\
\text { managing into the system. }\end{array}$ & $\begin{array}{l}\text { 1. Node level decision } \\
\text { 2. Less network traffic } \\
\text { 3. Co-operation from all } \\
\text { network nodes } \\
\text { 4. Not depend of single } \\
\text { node } \\
\text { 5. Less Storage required }\end{array}$ & $\begin{array}{l}\text { 1. Difficult to decide when to } \\
\text { start fault detection } \\
\text { 2. Malicious nodes effects } \\
\text { decisions }\end{array}$ \\
\hline
\end{tabular}




\begin{tabular}{|c|c|c|c|}
\hline Cluster & $\begin{array}{l}\text { The cluster based architecture } \\
\text { implemented fault detection } \\
\text { mechanism in a distributed manner } \\
\text { through intra cluster communication } \\
\text { and reports the failed nodes to the } \\
\text { upper layer of communication } \\
\text { hierarchy. }\end{array}$ & $\begin{array}{l}\text { 1. Combination of center } \\
\text { and distributed types } \\
\text { 2. Managing fault node is } \\
\text { easy } \\
\text { 3. No single node centric } \\
\text { 4. Allow node level } \\
\text { decision }\end{array}$ & $\begin{array}{l}\text { 1. Cluster head selection } \\
\text { 2. Hierarchical topology } \\
\text { required } \\
\text { 3. Different approach needed } \\
\text { to select fault in node and } \\
\text { cluster head. }\end{array}$ \\
\hline
\end{tabular}

\section{Proposed Work}

Proposed work supposed that each and every sensor nodes are arbitrarily spread in the network range as well as each sensor node is expected to identify its individual place and also its neighboring node and sink node too. Also all sensor nodes have the equal quantity of initial energy and equal maximum communication range. In this proposed scheme, every time due limited energy resources sensor nodes get fail to keep communicating with neighboring node and also link with destination node get break. This causes end of the network. In proposed scheme using self healing concept proposed routing protocol predict the fault which may cause due to node energy drain or node moving out of transmission range. Self healing system is set of algorithms to identify, and analyze the root cause of the faults (Network Fault Detection System) and then correct them using Fault Management System. Critical part of Self-healing is able to detect failure of a node and once detect those nodes then how re-organize network and recover from node failures without losing any information and controllable delay. Proposed work performance comparison done with existing Ad hoc On Demand Distance Vector (AODV) routing protocol. Proposed system increases throughput, decrease delay which verified and presented in performance analysis.

\section{Performance Analysis of Routing Protocol}

\subsection{Simulation Methodology and Parameters}

The planned method considered network simulator Ns3 for simulation. Simulation helps to recognize the actions and presentation of the network and its protocol. The proposed scheme considered node 0 as sink node and others are source nodes.

Table2. Simulation Parameters

\begin{tabular}{|c|c|}
\hline Parameters & Values \\
\hline Network Simulator & Ns3.20 \\
\hline Connection Type & UDP \\
\hline Simulation Time & $100 \mathrm{~ms}$ \\
\hline Number of Nodes & 120 \\
\hline Simulation Area & $500 \mathrm{~m} * 500 \mathrm{~m}$ \\
\hline Node Pause & $0 \mathrm{~s}$ \\
\hline Traffic Flow & CBR \\
\hline
\end{tabular}




\section{Result Analysis}

\subsection{Throughput}

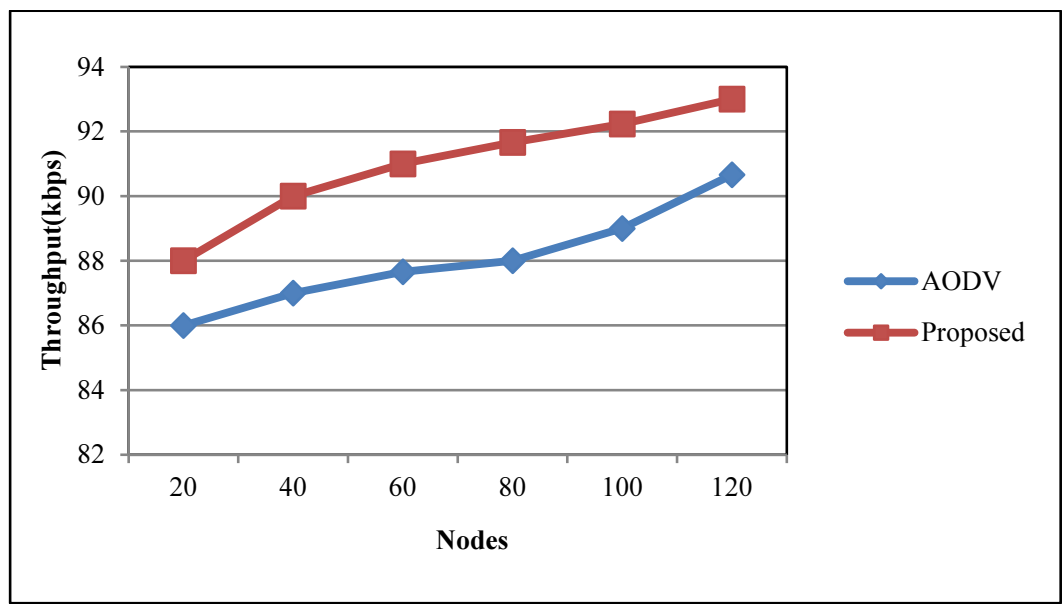

Figure1. Throughput Graph

The amount of successful message delivery from source node to destination node in the network is called throughput. Proposed protocol throughput is more than AODV. Fault management scheme handle the fault and reduces repeated path discovery.

\subsection{Delay}

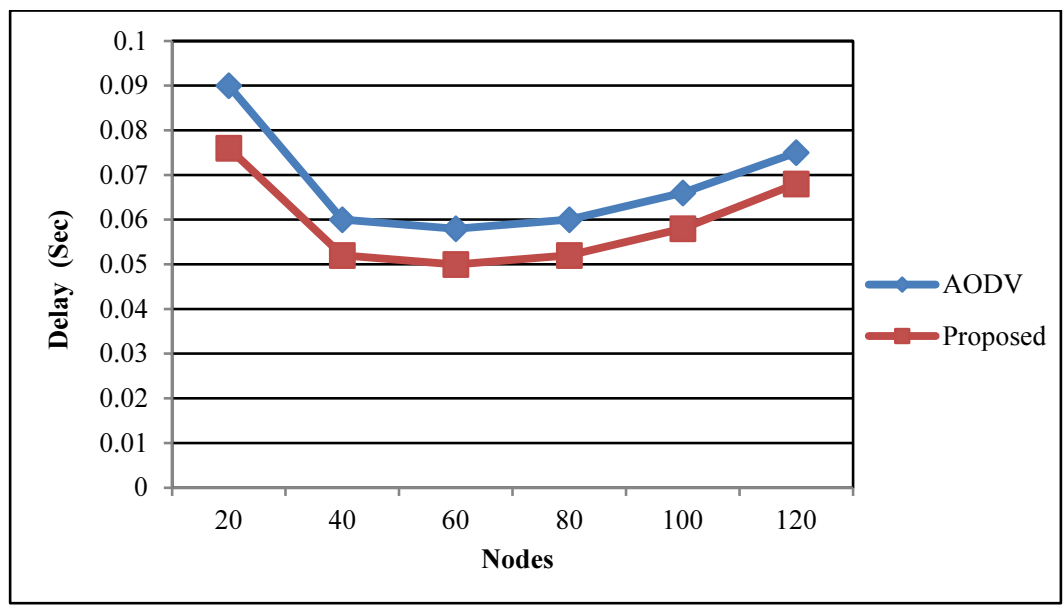

Figure2. Delay Graph

Delay means specifies the amount of time data the packets takes to reach the destination. Proposed scheme reduces delay than AODV routing protocol. 


\section{Conclusion}

Wireless sensor network is linked of numerous wireless detecting nodes. These tiny sensor devices have limitation of resource and randomly installed in harsh location. Hence, it is very common for wireless networks to occur faults, node failure, routing path or network fault etc. Proposed scheme, presented self healing fault managing scheme for wireless sensor network to detect mistakes and execute suitable methods to improve sensor network from failure. The proposed fault managing scheme is help to increase the performance of wireless sensor network. Comparative simulation result shows that proposed work improves the throughput. Main reason behind increase in throughput less link failure reduces packet drop and self healing avoids repeated path discovery leads to reduce in delay. Reduced delay and increase in packet delivery, resulted in better throughput.

\section{References}

[1] Pallavi Katkar, V. R. Ghorpade, A Survey on energy Efficient Routing Protocol for Wireless Sensor

[2] Networks, International Journal of Computer Science and Information Technology, Vol.6, 2015, 81-83

[3] V. Ranga, M. Dave, A. K. Verma, Node stability aware energy efficient single node failure recovery

[4] approach for WSANs, Malaysian Journal of Computer Science, Vol. 29, 2016, 106-123

[5] C. Zhu, C. Zheng, L. Shu, and G. Han, A survey on coverage and connectivity issues in wireless sensor networks, Journal of Network and Computer Applications, Vol. 35, 2012, 619-632

[6] I.F.Akyildiz, W.Su, Y.Shankarasubramaniam, E.Cayirci, Wirless sensor networks: a survey, Computer Network, Vol. 38, 2002, 393-422

[7] Jamal N. AL-Karaki, Ahmed E. Kamal, ROUTING TECHNIQUES IN WIRELESS SENSOR NETWORKS: A SURVEY, IEEE Wireless Communications, 2004

[8] Shahram Babaie, Ahmad Khadem-zadeh and Kambiz Badie, Distributed Fault Detection Method and Diagnosis of Fault Type in Clustered Wireless Sensor Networks, Life Science Journal, 2012

[9] Ting-Yu Lin, Hendro Agus Santoso, and Kun-Ru Wu, Global Sensor Deployment and Local Coverage-Aware Recovery Schemes for Smart Environments, IEEE Transactions on Mobile, $2014,14(7)$

[10] Hsung-Pin Chang and Tsung-Yu Yeh, A Software-based Fault Detection Scheme for Wireless Sensor Networks, IEEE International Conference on Computer and Information Science, 2016

[11] Gómez-Andrades, P. Muñoz, E. J. Khatib, I. de-la-Bandera, I. Serrano, and R. Barco, Methodology for the Design and Evaluation of Self-Healing LTE Networks, IEEE Transaction on Vehicular Technology, 2015, Vol. 65

[12] Pardeep Kumar Tanisha Saini, TO PROPOSE A TECHNIQUE FOR FAULT TOLERANCE IN WIRELESS SENSOR NETWORKS, International Journal of Computer Engineering and Technology, 2016, Vol. 7

[13] Rab Nawaz Jadoon, Adnan Anwar Awan, Muhammad Amir Khan, WuYang Zhou and Aamir Shahzad, An Efficient Nodes Failure Recovery Management Algorithm for Mobile Sensor Networks, Mathematical Problems in Engineering Hindawi, 2020

[14] Chiatanya Vijaykumar Mahamuni, Sensor Node Failure Affecting Coverage of WSNs: An Overview, International Journal of Emerging Technology in Computer Science and Electronics, 2015, Vol.14

[15] Rukhsar Begum Shaikh, Abid H Sayed, SENSOR NODE FAILURE DETECTION IN WIRELESS SENSOR NETWORK: A SURVEY, International Journal of Emerging Technology in Computer Science and Electronics, 2015, Vol. 14

[16] Elham Moridi, Majid Haghparast, Mehdi Hosseinzadeh, Somaye Jafarali Jassbi, Fault management frameworks in wireless sensor networks: A survey, Computer Communications ELSEVIER, 2020, Vol.155 